\title{
The structural basis of water permeation and proton exclusion in aquaporins (Review)
}

\author{
DAX FU \& MIN LU \\ Department of Biology, Brookhaven National Laboratory, Upton, New York, USA
}

(Received 2 February 2007; and in revised form 4 May 2007)

\begin{abstract}
Aquaporins (AQPs) represent a ubiquitous class of integral membrane proteins that play critical roles in cellular osmoregulations in microbes, plants and mammals. AQPs primarily function as water-conducting channels, whereas members of a sub-class of AQPs, termed aquaglyceroporins, are permeable to small neutral solutes such as glycerol. While AQPs facilitate transmembrane permeation of water and/or small neutral solutes, they preclude the conduction of protons. Consequently, openings of AQP channels allow rapid water diffusion down an osmotic gradient without dissipating electrochemical potentials. Molecular structures of AQPs portray unique features that define the two central functions of AQP channels: effective water permeation and strict proton exclusion. This review describes AQP structures known to date and discusses the mechanisms underlying water permeation, proton exclusion and water permeability regulation.
\end{abstract}

Keywords: Aquaporin, protein structure, mechanisms underlying water permeation, membrane protein, membrane permeability

\section{Introduction}

Water can diffuse freely through the lipid bilayer at a limited rate, but it was noted that water permeability in red blood cells was much higher than expected for spontaneous diffusion. This observation prompted the search for specialized proteins that may exist to facilitate water fluxes across membranes. The putative water channel from the red blood cell membrane was initially purified as a $28 \mathrm{kDa}$ membrane-bound species, named Channel-forming Integral Protein of $28 \mathrm{kDa}$ (CHIP28) (Denker et al. 1988). Expression of CHIP28 in Xenopus oocytes was later shown to induce rapid water diffusion down osmotic gradients across the plasma membrane, leading to the identification of the first water-conducting channel (Preston et al. 1992). Protein sequence analysis suggested that CHIP28 is homologous to Major Intrinsic Protein (MIP) from the lens fiber cells (Gorin et al. 1984). Shortly after establishing the water pore function of CHIP28, homologous proteins in mammals, bacteria and plants were found to function as water channels, and the term 'aquaporin' was coined for members of this protein family (Agre, Sasaki \& Chrispeels 1993). Accordingly, CHIP28 was renamed to AQP1 and MIP is now known as AQP0.
Since the discovery of AQP1, several hundred $A Q P$ homologues have been identified from all kingdoms of life, including 13 mammalian AQPs (Yasui, 2004, Ishibashi 2006). Based on the permeability property of each AQP, mammalian homologues are loosely clustered into two subsets: aquaporins and aquaglyceroporins. The eubacterium Escherichia coli offers a model system for this categorization, as it contains two aquaporin paralogues: a water-conducting aquaporin AqpZ, and an aquaglyceroporin GlpF that is permeable to both glycerol and water (Borgnia \& Agre 2001). Most members from the first category (aquaporins) are only permeated by water, which includes AQP0, AQP1, AQP2, AQP4 and AQP5 (Agre et al. 1993). The inclusion of AQP6 and AQP8 into the first category is, on the other hand, primarily based on sequence homology, since AQP6 is also permeated by anions (Yasui et al. 1999), and AQP8 perhaps by both water and urea (Ishibashi et al. 1997). Members of the second group (aquaglyceroporins), which includes AQP3, AQP7, AQP9 and AQP10, are permeable to small neutral solutes, such as glycerol and urea, with or without water co-permeation (Hara-Chikuma \& Verkman 2006). Among them, AQP9 exhibits the broadest substrate specificity

Correspondence: Dax Fu, Department of Biology, Brookhaven National Laboratory, Upton, NY 11973, USA. Tel: +1 6313444208. E-mail: dax@bnl.gov 
(Tsukaguchi et al. 1998). The substrate specificities for the recently identified AQP11 and AQP12 (Morishita et al., 2004, Gorelick et al. 2006) remain to be determined. Some archaeabacterial AQPs may form a third subfamily that lies somewhere between aquaporins and aquaglyceroporins (Kozono et al. 2003). In plants, there are a remarkably large number of AQP genes (Johanson et al. 2001), which fall into four subfamilies (Borstlap 2002), PIPs (Plasma membrane Intrinsic Proteins), TIPs (Tonoplast Intrinsic Proteins), NIPs (NOD26-like Intrinsic Proteins) and SIPs (Small basic Intrinsic Proteins). The TIPs show a range of water conductivities from high to moderate, while the PIPs have moderate to minimal water conductivities (Kaldenhoff \& Fischer 2006).

Hydropathy analysis of AQP primary sequences predicted that AQPs contains six membrane-spanning segments (TM1-6) with five connecting loops (A-E) (Smith \& Agre 1991) (Figure 1B). The membrane topology of AQP1 was established by

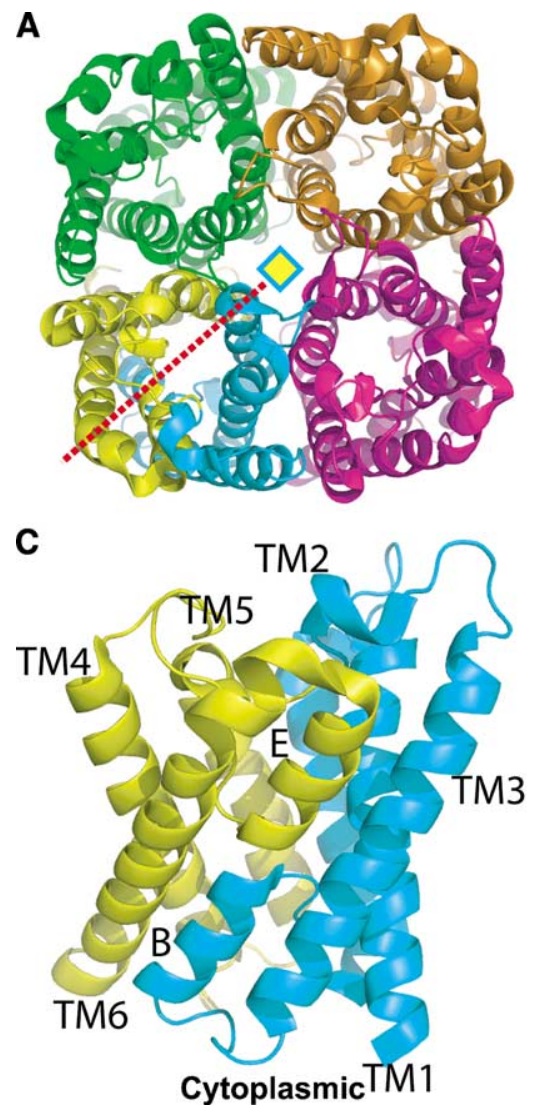

epitope-scanning mutagenesis and vectorial proteolysis, showing that both $\mathrm{N}$ - and C-termini are located in the cytoplasm (Preston et al. 1994). Interestingly, the $\mathrm{N}$ - and C-terminal halves of AQPs share about $20 \%$ sequence identity. This genetic relation suggests that the AQP family arose by tandem gene duplication during evolution (Pao et al. 1991). The cytoplasmic loop B and the extracellular loop $\mathrm{E}$ are highly related to each other, with each containing a tripeptide signature motif asparagine, proline, alanine (NPA). Since these two loops are rather hydrophobic in nature, it was proposed that they are structural components of the aqueous pore (Jung et al. 1994).

One remarkable feature of AQP1 is the lack of detectable proton conductivity despite massive water permeability (Zeidel et al., 1992, Pohl et al. 2001; Saparov et al. 2005). Rapid water diffusion in AQP channels would necessitate the line-up of multiple water molecules in a single file with each water molecule hydrogen-bonded to its neighbors. As
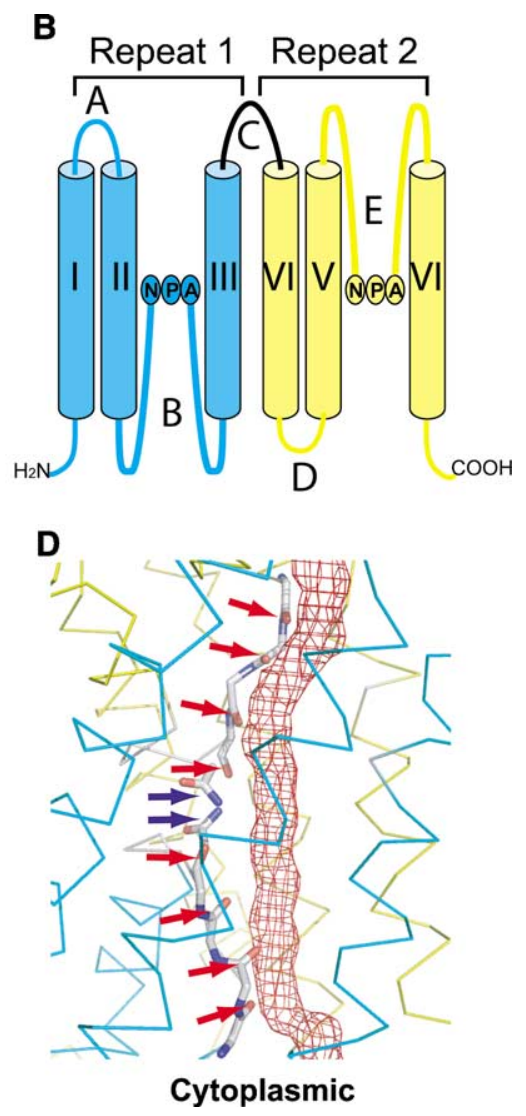

Figure 1. (A) Ribbon representation of AqpZ tetramer, viewed from the periplasmic side down the tetramer four-fold axis (diamond). One monomer is colored in both cyan and yellow, representing the $\mathrm{N}$ - and $\mathrm{C}$-terminal halves that are related by a quasi-two-fold axis (red dashed line). (B) Membrane topology of AQP. Two genetic repeats (Repeat-1 and Repeat-2) are colored in cyan and yellow respectively. Membrane-spanning helices are denoted as I-VI, loops A-E, and the NPA signature sequences are labeled. (C) AqpZ monomer viewed from the membrane plane. Two repeats are colored and transmembrane spanning helices labeled as above. (D) Pore-lining carbonyl oxygens and asparagine amines in AQP1. The boundary of the water-conducting channel, as estimated by HOLE (Smart, Goodfellow \& Wallace 1993), is represented as a red meshed tube. Two patches of carbonyl oxygens and two central asparagine amines are indicated by red and blue arrows respectively. All figures were rendered with PyMol. 
such, protons would rapidly hop along a string of water molecules, resulting in proton leakage (Pomes \& Roux, 1996). In virtually all organisms, proton gradients across cellular and subcellular membranes are the primary energy source for ATP synthesis. A proton leakage accompanied with water flux through aquaporins could be potentially lethal. It has been a long-standing puzzle as to how efficient water permeation and strict proton exclusion can be reconciled in AQPs. In recent years, a significant progress has been made in structural analysis of AQPs by both electron and x-ray crystallography (Gonen \& Walz 2006). At present, atomic models of seven AQPs are available, including red blood cell AQP1 (Murata et al. 2000, Ren et al. 2001, Sui et al. 2001), E. coli glycerol facilitator $\mathrm{GlpF}$ (Fu et al. 2000, Tajkhorshid et al. 2002), E. coli water pore AqpZ (Savage et al. 2003, Jiang et al. 2006), eye lens-specific AQP0 (Gonen et al. 2004, Harries et al. 2004), archaeabacterial AqpM (Lee et al. 2005), spinach aquaporin SOPIP2;1 (Tornroth-Horsefield et al. 2006), and AQP4 from brain (Hiroaki et al. 2006). These AQP structures provide the basis for mutation-function analysis and atomistic computer simulations, leading to significant insights into the mechanisms of water permeation and proton exclusion.

\section{Overall architecture}

Despite the evolutionary distance from bacteria to humans, transmembrane spanning domains of human aquaporin AQP1 and $E$. coli aquaglyceroporin GlpF can be superimposed with a root-mean-square deviation of $1.8 \AA$ for backbone atoms. All known AQP structures exhibit a common homo-tetrameric arrangement (Figure 1A), and each AQP monomer is folded into a right-handed alpha-barrel architecture, with a central transmembrane channel surrounded by six full length transmembrane helices and two NPA-containing loops, $\mathrm{B}$ and $\mathrm{E}$ (Murata et al. 2000) (Figure 1C). These loops project into the center of the membrane as extended polypeptide chains and return to the same side of the membrane surface as half-spanning helices, which are joined at their N-terminal ends near the center of the membrane plane by interlocking interactions between two NPA motifs. AQP monomer structures are characterized by an internal quasi-two-fold symmetry, corresponding to the intragenic gene duplication of AQP sequences. The quasi-two-fold axis lies in the membrane plane, running through the NPA junction and intersecting the tetramer four-fold axis. The two halves of AQPs reiterate an identical protein fold, but with inverted transmembrane orientations (Figure 1C). Constrained by the internal symmetry, all inter-helix contacts in AQPs are coplanar with the quasi-two-fold axis in the membrane plane (Fu et al. 2000). Transmembrane helices diverge outwards from the membrane plane to yield a characteristic hourglass shaped structure.

The hourglass-shaped structure of AQPs has an extracellular and a cytoplasmic vestibule where waters are present as a bulk solvent. These vestibules are connected by a central amphipathic pore 20-25 $\AA$ in length. The hydrophilic face of the amphipathic pore provides two patches of solvent-accessible mainchain carbonyls, each contributed by the extended polypeptide chain of loop E from the extracellular side and the quasi-2-fold related loop B from the cytoplasmic side. Between these mainchain carbonyls are the highly constrained NPA junction, where two asparagines project their sidechains to the channel. This complete set of pore-lining carbonyl oxygens and asparagine amines forms a contiguous array of hydrogen bond acceptors and donors for coordination of water and/or glycerol molecules (Figure 1D). On the hydrophobic face of the channel, an abundance of hydrophobic residues set steric limits to the size of the pore. For a water or glycerol molecule to enter this narrow pore from the bulk solvent, its hydration shell has to be stripped off before it can 'squeeze' through the pore. The hydrogen bond donor and acceptor groups on the hydrophilic face serve as surrogate waters that offer replacement interactions to compensate for the energetic cost of dehydration (de Groot \& Grubmuller 2001, Tajkhorshid et al. 2002).

X-ray structures of AQP1 and the glycerol facilitator GlpF enabled molecular dynamics simulations of spontaneous, full permeation events in aquaporins (de Groot \& Grubmuller 2005). It was found that both AQP1 and GlpF act as two-stage filters, corresponding to the NPA junction in the midmembrane plane and a selectivity filter located approximately $10 \AA$ away from the NPA junction on the periplasmic side ( $\mathrm{Fu}$ et al. 2000). The selectivity filter was also known as the aromatic/ arginine ( $\mathrm{ar} / \mathrm{R})$ constriction, because it contains conserved aromatic and arginine residues (Sui et al. 2001). The ar/R constrictions of AQP1 and $\mathrm{GlpF}$ are located at the narrowest point of the channels. Different sidechains at the ar/R constriction influence the polarity and diameter of the channel bottleneck, thereby separating aquaporins from aquaglyceroporins (Savage et al. 2003). On the other hand, the invariant NPA constriction is vital for proton exclusion, a feature common to all AQPs (Murata et al. 2000). In the next section, the structural features of these constrictions, together with results of molecular dynamics simulations and mutation-function analysis will be discussed. 


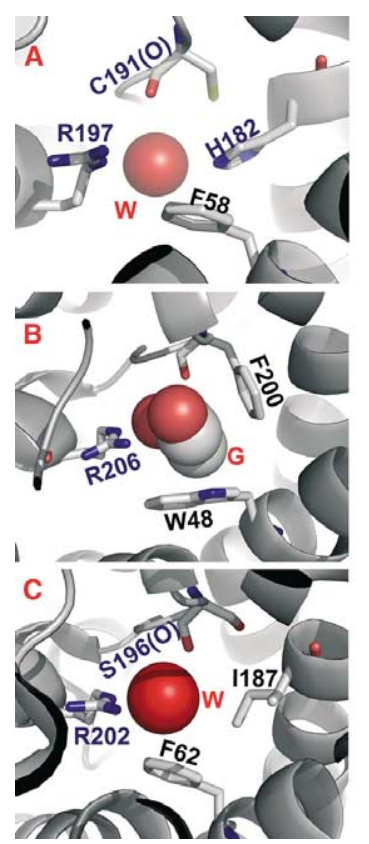

D
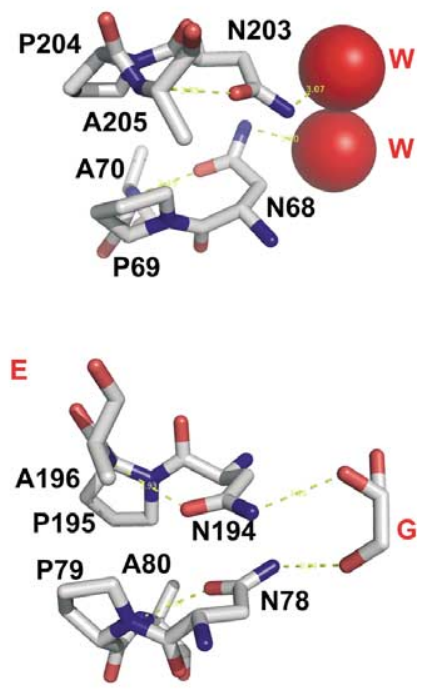

Figure 2. (A-C) ar/ $\mathrm{R}$ constriction sites of AQP1 (A), GlpF (B) and AqpM (C), viewed from the extracellular side down the channel. A water (W) or glycerol (G) molecule is bound at the center of the ar/ $\mathrm{R}$ constriction with coordination residues labeled. D-E, NPA junctions of AQP1 (D) and GlpF (E) with coordinated waters (W) or glycerol (G). Dashed lines indicate hydrogen bonds.

\section{Ar/R constriction and water/glycerol selectivity}

The ar/R constriction in AQP1 is approximately 2.8 $\AA$ in diameter, formed by R197, H182, F58 and C191 (Sui et al. 2001) (Figure 2A). Residues H182 and R197, along with the solvent-accessible C191 carbonyl, constitute the hydrophilic face of the constriction. Opposite to the hydrophilic face lies residue F58 that defines the hydrophobic face. The presence of C191 explains mercurial-inhibition of AQP1 water-permeability (Preston et al. 1993). R197, H182 and F58 are conserved among the water-specific aquaporins (Park \& Saier 1996). A water molecule was found hydrogen-bonded to the NE2 nitrogen of H182 and the G192 carbonyl adjacent to the constriction region.

In AqpZ, the ar/R constriction is formed by the sidechains of R189, H174 and the carbonyl of T183 on the hydrophilic face, and the aromatic ring of F43 on the hydrophobic face (Savage et al. 2003) (Figure 3A). The AqpZ and AQP1 ar/R constriction sites are nearly identical, with the exception that the AQP1 C191 is substituted for the AqpZ T183. In a more recent crystal structure of tetramer AqpZ, R189 adopts distinct conformations in different protomers (Jiang et al. 2006). In one protomer, the R189 guanidinium points toward the periplasmic vestibule, opening up the constriction to accommodate the binding of a water molecule (Figure 3A). Whereas in other protomers, the

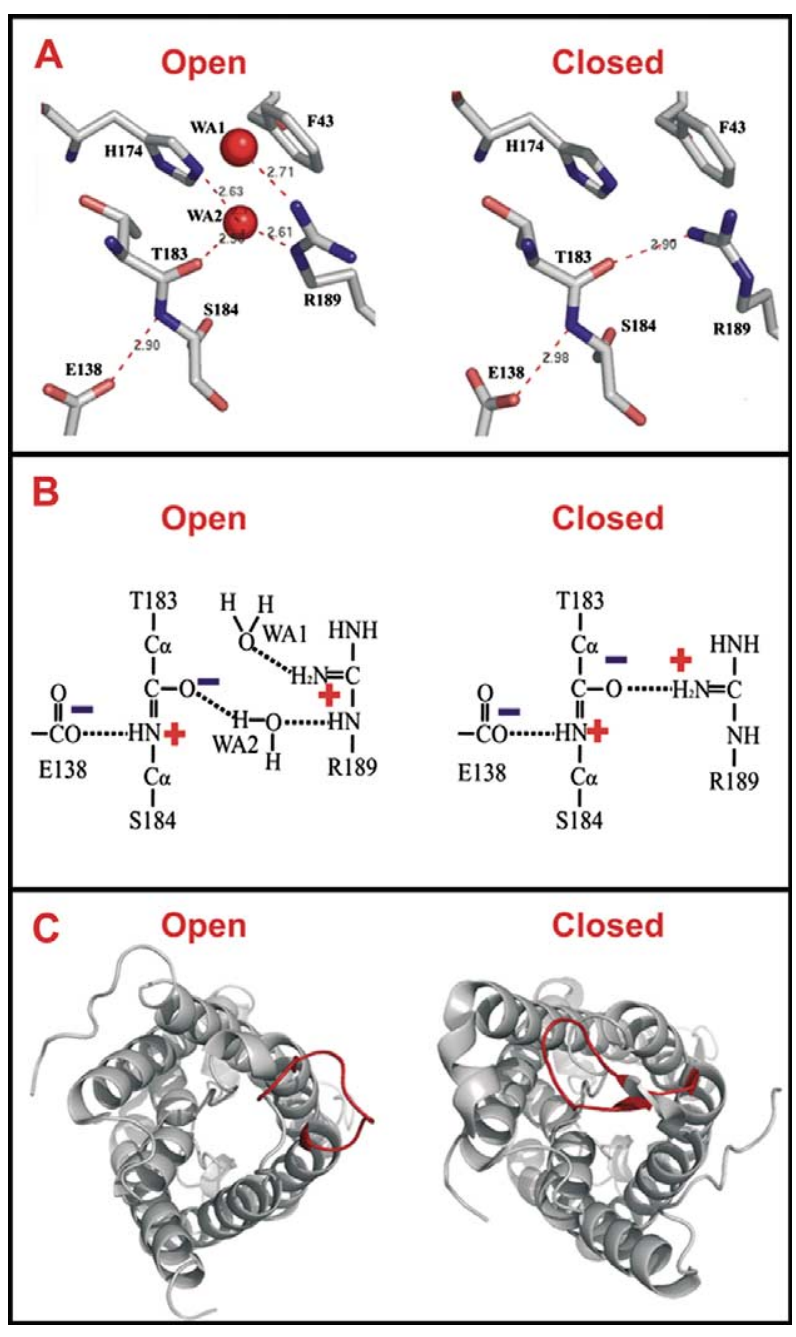

Figure 3. (A) Hydrogen bond network in the ar/R constriction of an open or closed AqpZ channel. (B) The electrostatic interaction between R189 and E138 and its contribution to the dual polarization of the bound water molecule (Jiang et al. 2006). (C) Open and closed conformations of the SoPIP2;1 structure, viewed from the cytoplasmic side down the water channel. The major difference between these two conformations is the displacement of the cytoplasmic loop D (red) that blocks the channel entrance in the closed conformation.

R189 guanidinium bends over to form a hydrogenbond with the carbonyl of T183, thereby occluding the channel. This observation is consistent with two distinct R189 conformations predicted by molecular dynamics calculations (Wang et al. 2005).

Of all the AQP structures, only the aquaglyceroporin GlpF has a glycerol molecule bound at the ar/ $\mathrm{R}$ constriction site, which is barely large enough in cross section to accommodate a single $\mathrm{CH}-\mathrm{OH}$ group of a glycerol molecule (Fu et al. 2000) (Figure 2B). This ar/R constriction is strongly amphipathic, with the planes of two perpendicularly arranged aromatic rings (W48 and F200) forming a hydrophobic corner that packs against the alkyl backbone of the bound glycerol. The guanidinium group of 
R206 and the carbonyl oxygens of G199 and F200 on the opposing hydrophilic face provide hydrogen bond partners for interactions with a pair of glycerol hydroxyls. The third glycerol hydroxyl is hydrogenbonded to a water molecule, allowing one-to-one stoichiometric co-permeation of glycerol and water. Compared with the $\mathrm{ar} / \mathrm{R}$ constriction of the waterspecific AqpZ, only the arginine is invariant, whereas F43, H174 and T183 of AqpZ are replaced by W48, G191 and F200 in GlpF, respectively. The $\mathrm{H} \rightarrow \mathrm{G}$ substitution provides room needed to accommodate additional $\mathrm{F} \rightarrow \mathrm{W}$ and $\mathrm{T} \rightarrow \mathrm{F}$ substitutions, resulting in increases of both size and hydrophobicity of the $\mathrm{GlpF}$ ar/R constriction. Although the resulting constriction site has a larger diameter of $3.8 \AA$, water permeability in GlpF is substantially lower than those measured for AqpZ and AQP1 (Borgnia \& Agre 2001). It appears that the more hydrophobic nature of the $\mathrm{GlpF}$ ar/R constriction contributes to lower water permeability.

AQP0 is present as a $26 \mathrm{kDa}$ full-length protein in young lens fiber cells, but upon aging it undergoes proteolysis to a $22-\mathrm{kDa}$ form (Roy, Spector \& Farnsworth 1979). This cleavage has been shown to induce junction formation between lens fiber cells (Kistler \& Bullivant 1980, Zampighi et al. 1989). Both junctional (truncated) and isolated (fulllength) AQP0 structures have been determined by electron and X-ray crystallography, respectively (Gonen et al. 2004, Harries et al. 2004). A comparison of the two AQP0 structures, however, reveals no global structural difference (Harries et al. 2004). The junctional AQP0 ar/R site is formed by residues R187, H172, F48 and A181. Substitution of AQP1 C191 with AQP0 A181 renders AQP0 resistance to mercurials. R187 forms a hydrogen bond with a backbone carbonyl, adopting a conformation similar to the closed ar/ $\mathrm{R}$ constriction site observed in AqpZ (Jiang et al. 2006). This R187 conformation, in combination with additional tyrosine constrictions and an extended ar/ $R$ constriction found in the junctional $\mathrm{AQP} 0$, provides the basis of limited water permeability in AQP0 (see below).

The ar/ $\mathrm{R}$ constriction in AqpM is formed by R202, F62, I187 and the carbonyl of S196 (Figure 2C). The major distinction of AqpM is made by I187, which replaces a histidine in AQP1, AqpZ and AQP0 (Lee et al. 2005). This histidine residue seems critical in establishing water specificity, since it is conserved among all known water-specific AQPs (Sui et al. 2001). In AQP1, H182 provides a hydrogen-bond for a water molecule. The $\mathrm{H} \rightarrow \mathrm{I}$ substitution in AqpM removes the hydrogen-bond donor. Perhaps for this reason, AqpM conducts water at a lower rate than AqpZ and AQP1 (Kozono et al. 2003). A secondary effect of the $\mathrm{H} \rightarrow \mathrm{I}$ substitution would result in a slightly wider and more hydrophobic ar $/ \mathrm{R}$ constriction. This change may adapt AqpM to conduction of larger and less polar solutes. Indeed, in vitro studies have demonstrated a limited glycerol conductance in AqpM (Kozono et al. 2003). The AqpM structure reveals four water molecules and three glycerol molecules lined up in a single file along the channel, but only a water molecule bound at the $\operatorname{ar} / \mathrm{R}$ constriction. Thus, the AqpM ar/R constriction seems to resemble those of water-specific aquaporins (Lee et al. 2005).

Structural analyses of various AQPs suggest that both size and polarity of the ar/R constriction define water/glycerol selectivity and permeability. However, a recent mutational analysis provided a more convoluted picture. For examples, AQP1 structure predicted that substituting the histidine or arginine at the $\mathrm{ar} / \mathrm{R}$ constriction with hydrophobic residues would hinder water isolation from its bulk solvent, thus reducing water permeability (Sui et al. 2001). Surprisingly, even a H180A/R195V double mutation did not alter water permeability (Beitz et al. 2006). The expected polarity decrease may be compensated for by a size increase at the ar/ $\mathrm{R}$ constriction. In line with the size effect, the H180A/R195V double mutation rendered AQP1 permeable to bulky molecules like urea. Further, increasing the diameter of the ar/R constriction by the F56 and H180 mutations conferred glycerol and urea permeabilities (Beitz et al. 2006). However, a size effect alone couldn't account for selectivity against ammonia in AQP1, because ammonia has a size and dipole moment similar to those of water. Ammonia permeability was found to correlate with the polarity of ar/ $\mathrm{R}$ constriction sites of various AQP1 mutants, suggesting that certain hydrophobic niches at the $\mathrm{ar} / \mathrm{R}$ constriction are needed for ammonia passage (Beitz et al. 2006). It appears that the interplay of both size and polarity at the ar/R constriction site plays a major role in defining channel selectivity and permeability.

Recent mutational studies have also demonstrated that removal of the positive charge from the ar $/ R$ constriction can cause proton leakage through the AQP1 pore (Beitz et al. 2006). The electrostatic proton barrier in AQPs thus involves not only the NPA motifs (see below), but also the ar/R constriction, where the positive charge on the highly conserved arginine may discourage the approaching of protons. In addition, at this narrowest point of the channel, the arginine forms a strong electrostatic interaction with a backbone carbonyl (Left panel, Figure $3 \mathrm{~A}$ ). This carbonyl is expected to be highly polarized due to the resonance of the mainchain peptide bond under the influence of a fully buried 
glutamate carboxylate (Jiang et al. 2006). Thus the partial negative charge on the carbonyl may provide the basis of anion exclusion. Furthermore, the carbonyl at the ar/ $R$ constriction of AqpZ is only 3-5 angstroms apart from the guanidinium $\mathrm{NHs}$, depending on the arginine conformations. The close proximity of a pair of charges in the highly restrictive ar/ $\mathrm{R}$ constriction site reinforces the interplay between geometry and polarity. It appears that the $\mathrm{ar} / \mathrm{R}$ constriction only permits the passage of dipoles that can interact with positive and negative charges simultaneously. Both cations and anions are excluded because neither can fulfill a dual role of polarization (Fu et al. 2002). It is evident that both water and glycerol can satisfy the polarity and geometry requirements of the ar/ $\mathrm{R}$ constrictions. In AqpZ, a water molecule acts as a hydrogen bond acceptor from R189 NE and a donor to T183 carbonyl at the same time (Jiang et al. 2006) (Right panel, Figure 3B). Likewise, in GlpF, two successive $\mathrm{OH}$ groups of a glycerol molecule are dually hydrogen-bonded to R206 NH2 and NE and carbonyls of G199 and F200 (Fu et al. 2000). An exception to this rule may be provided by AQP6, which conducts anions at low $\mathrm{pH}(<5.5)$. At this $\mathrm{pH}$, protonation of the polarized carbonyls at the ar/R constriction site may weaken the negative electrostatic repulsion, thereby lowering the electrostatic barrier against anion passage. However, whether the ar/ $\mathrm{R}$ constriction of AQP6 is directly involved in the observed anion conductance has yet to be established.

\section{NPA junction and proton exclusion}

In AQP channels, the two half-spanning helices are capped by NPA motifs that are held together about the quasi-twofold-axis in the membrane plane (Figure 2D). This NPA junction is formed by Van der Waals contacts of the proline rings and an interlocking hydrogen-bond network that orients asparagine sidechains and projects their terminal amine groups into the pore. In AQP1, two waters are centered about the NPA junction, each being hydrogen-bonded to the ND2 groups of a NPA asparagine (Sui et al. 2001) (Figure 2D). In AqpZ, two NPA asparagine ND2 groups function as hydrogen-bond donors to a central water molecule, locking it in a conformation in which this water can only donate hydrogen bonds, but can not accept one from adjacent waters (Savage et al. 2003). In AQP0, the channel near the NPA junction is significantly narrower, restricted in between a pair of tyrosine constriction sites (see below) (Harries et al. 2004). In both GlpF and AqpM, the NPA junctions present a pair of hydrogen-bond donors to successive hydroxyls of a bound glycerol mole- cule (Figure 2E). Interestingly, only glycerol was found at the NPA junction of GlpF, but not AqpZ, although both proteins were crystallized in high glycerol concentrations. It appears that the local environments around the NPA junction may contribute to this difference in water/glycerol coordination (Savage et al. 2003).

The crystal structure of AQP1 revealed a single file of six water molecules in the pore (Sui et al. 2001), and the GlpF structure determined in the absence of glycerol contained nine water molecules (Tajkhorshid et al. 2002). Similarly, the AqpZ structure contained five water molecules in each pore (Savage et al. 2003), and the isolated AQP0 structure contained seven water molecules (Harries et al. 2004). These water molecules could align themselves in a long chain, in effect forming a wire that would allow protons to jump between neighboring waters (Bernal 1965). It is remarkable that aquaporins, which inherently contain a line of water molecules, could preserve the proton electrochemical gradient. The head-to-head arrangement of two half-spanning helices point their positive ends towards the membrane plane, generating net positive charges in the middle of the AQP channel (Murata et al. 2000). A water molecule at the NPA junction may orient itself so that its oxygen atom forms hydrogen bonds with two asparagine amines, losing its capacity for accepting protons from neighboring waters. This water conformation, together with the positive dipole charges, was proposed to disrupt proton conduction along a continuous single file of waters in AQP channels (de Groot \& Grubmuller 2001).

Molecular dynamics/quantum mechanical simulations of water molecules in AQP1 and GlpF suggested that the central water is nevertheless capable of forming hydrogen bonds with the neighboring water molecules, but it can only engage in hydrogen bonds orienting outwards from the NPA junction towards the extracellular and the cytoplasmic entrances of the channel (de Groot \& Grubmuller 2001, Tajkhorshid et al. 2002). Therefore, the lines of water molecules in the two halves of the channel have opposite hydrogen-bond polarity, preventing protons from crossing the central water but allowing water to permeate (Tajkhorshid et al. 2002). In support of this model, orientations of water molecules observed in AqpZ crystal structures indeed are ordered in a bipolar manner, with oxygen atoms facing the NPA junction in the center of the channel (Savage et al. 2003, Jiang et al. 2006). However, a recent simulation study suggested that the proton wire is actually fully intact throughout the water channel, but the protons could not overcome the electrostatic barrier imposed by the helical 
dipoles at the NPA junction (de Groot et al. 2003). It appears that unfavorable electrostatic interactions, rather than proton wire interrupting effects, are the primary reason for proton exclusion (Chakrabarti et al. 2004). The bipolar water orientation, on the other hand, is merely a secondary effect of such electrostatic interactions (de Groot \& Grubmuller 2005).

\section{Additional constrictions and regulation of water permeability}

The water permeability of AQP0 is about 40 times lower than that of AQP1 (Chandy et al. 1997). In addition to the ar/R and NPA constrictions, both isolated and junctional AQP0s have a pair of quasitwo-fold related tyrosine constrictions on either side of the NPA junction, with the narrowest constriction point relocated from their $\mathrm{ar} / \mathrm{R}$ sites to one of the tyrosine-constrictions close to the cytoplasmic entrance (Harries et al. 2004). These two tyrosines are fully conserved among all known AQP0s, reflecting their importance in restricting water permeability of AQP0. The main difference between the junctional and isolated AQP0 conformation lies in the orientation of M176 of the junctional AQP0, which obstructs the water pore from the extracellular side of the ar/ $\mathrm{R}$ constriction (Gonen et al. 2005). The ar/ $\mathrm{R}$ constriction of the isolated AQP0 opens up sharply on the extracellular side. This difference may account for a greater number of ordered water molecules found in the water-conducting channel of the isolated AQP0.

AQP0 water permeability is sensitive to $\mathrm{pH}$ (Hara-Chikuma \& Verkman 2006). Two histidine residues ( $\mathrm{H} 40$ and $\mathrm{H} 66)$ were implicated in $\mathrm{pH}$ dependence of AQP0 water conductance. The double-layered 2D crystals were grown at $\mathrm{pH} 6$ (Gonen et al. 2004), while the 3D crystals were obtained at $\mathrm{pH} 10.5$ (Harries et al. 2004). However, a comparison of these two structures provided no clues as to the structural consequence of $\mathrm{pH}$ variation that was expected to produce a 3 to 4 -fold difference in water permeability (Harries et al. 2004). The structural basis of $\mathrm{pH}$ regulation of AQP0 remains unknown.

Plant SoPIP2;1 channel is a gated AQP in response to water availability. For examples, drought stress causes channel closure by dephosphorylation of conserved S1 15 and S274 (Johansson et al. 1998), while flooding-induced anoxia decreases cytoplasmic $\mathrm{pH}$ and shuts off the water channel through protonation of H193 (Tournaire-Roux et al. 2003). The gating of water channels allows land plants to cope with rapid changes in the water availability. The structures of spinach SoPIP2;1 and a His-tagged variant both exhibit a typical water-specific $A Q P$ structure, with a continuous chain of seven water molecules running along the length of the channel (Tornroth-Horsefield et al. 2006). However, a major difference between these two structures, and perhaps with other AQPs, is the conformation of the cytoplasmic loop D (Figure 3C). In the SoPIP2;1 structure, the loop $\mathrm{D}$ is anchored to the $\mathrm{N}$ terminus by a hydrogen-bond network involving a divalent cation $\left(\mathrm{Ca}^{2+}\right)$. Residues L197 in loop D blocks the channel entrance from the cytoplasm and forms a hydrophobic barrier with H99, V104 and L108. Thus, this structure represents a closed conformation (Left, Figure 3C). It is noteworthy that L197 of SoPIP2; 1 in the closed conformation overlays almost exactly with Y149 of AQP0. In the structure of the His-tagged SoPIP2;1, the loop D is detached from the $\mathrm{N}$-terminus, and the $\mathrm{N}$-terminus of helix 5 extends a half-turn into the cytoplasm, resulting in the displacement of L197 away from the pore entrance (Right, Figure 3C). Since these structural changes open up the hydrophobic gate, the Histagged structure was proposed to portray an open conformation.

A comparison of these two conformations suggested that phosphorylation of S115 in the cytosolic loop B disrupts its anchoring interactions with the $\mathrm{N}$-terminus while phosphorylation of S274 may detach the C-terminus from its interactions with adjacent subunit in the tetramer, making room for the cytoplasmic end of helix 5 to extend an additional half-turn. The combined actions of S115 and S274 may trigger the movement of loop $\mathrm{D}$, leading to the opening of the hydrophobic gate. The proposed mechanism of pore opening was supported by molecular dynamics simulations (Tornroth-Horsefield et al. 2006). A mechanism of $\mathrm{pH}$-regulated PIP gating is also apparent from the closed structure of SoPIP2:1. A rotamer of $\mathrm{H} 193$ was proposed to form a salt bridge with D28 upon protonation (Tornroth-Horsefield et al. 2006). This would recover the anchor for loop $\mathrm{D}$ on the $\mathrm{N}$ terminus, which is lost upon phosphorylation of S115 and would thus result in the same closed pore conformation observed in the crystal structure (Tornroth-Horsefield et al. 2006). It is interesting to note that phosphorylation and $\mathrm{pH}$ are distinct chemical signals, acting on residues (S115, S274 and H193) well separated in sequence, but their actions converge to altering the conformation of loop D. This gating mechanism explains how plants respond to draught stress and flooding with an identical physiological response (Tornroth-Horsefield et al. 2006). 


\section{Further studies}

The structures of various aquaporins have provided significant insights into the molecular mechanisms of water/glycerol permeation and proton exclusion. Some members of the AQP family have evolved to permeate glycerol, linear polyols, nitrate, arsenite, gas or even anions (Heller, Lin \& Wilson 1980, Prasad et al. 1998, Yasui et al. 1999, Ikeda et al. 2002, Liu et al. 2002, Wallace \& Roberts 2005, Saparov et al. 2007). The structural determinants for this broad spectrum of permeants have yet to be characterized in full. Elucidation of the structural basis of channel selectivity would facilitate the design and discovery of AQP-modulating agents (Detmers et al. 2006). AQP water conductance can be regulated by a variety of chemical signals, including hormones, phosphorylation, intercellular $\mathrm{pH}$ and calcium levels (Nielsen et al. 2002, Gunnarson, Zelenina \& Aperia 2004, Chaumont, Moshelion \& Daniels 2005, Hedfalk et al. 2006). Regulatory components in AQP structures are largely unknown, and how a highly conserved AQP structural framework has evolved to meet various regulatory requirements remains to be explored. As more AQP structures become available, along with concerted efforts of mutagenesis analysis and dynamics simulations, AQPs will continue to offer an exciting model system for understanding fundamental principles that govern channel selectivity, permeability and gating.

\section{Acknowledgements}

This work was supported by a National Institute of Health grant RO1 GM65137 (to D. Fu). BNL is managed by Brookhaven Science Associates for the US Department of Energy.

\section{References}

Agre P, Sasaki S, Chrispeels MJ. 1993. Aquaporins: a family of water channel proteins. Am J Physiol 265:F461.

Beitz E, Wu B, Holm LM, Schultz JE, Zeuthen T. 2006. Point mutations in the aromatic/arginine region in aquaporin 1 allow passage of urea, glycerol, ammonia, and protons. Proc Natl Acad Sci USA 103:269-274.

Bernal JD. 1965. The structure of water and its biological implications. Symp Soc Exp Biol 19:17-32.

Borgnia MJ, Agre P. 2001. Reconstitution and functional comparison of purified GlpF and AqpZ, the glycerol and water channels from Escherichia coli. Proc Natl Acad Sci USA 98:2888-2893.

Borstlap AC. 2002. Early diversification of plant aquaporins. Trends Plant Sci 7:529-530.

Chakrabarti N, Roux B, Pomes R. 2004. Structural determinants of proton blockage in aquaporins. J Mol Biol 343:493-510.

Chandy G, Zampighi GA, Kreman M, Hall JE. 1997. Comparison of the water transporting properties of MIP and AQP1. J Membr Biol 159:29-39.
Chaumont F, Moshelion M, Daniels MJ. 2005. Regulation of plant aquaporin activity. Biol Cell 97:749-764.

de Groot BL, Frigato T, Helms V, Grubmuller H. 2003. The mechanism of proton exclusion in the aquaporin-1 water channel. J Mol Biol 333:279-293.

de Groot BL, Grubmuller H. 2001. Water permeation across biological membranes: mechanism and dynamics of aquaporin1 and GlpF. Science 294:2353-2357.

de Groot BL, Grubmuller H. 2005. The dynamics and energetics of water permeation and proton exclusion in aquaporins. Curr Opin Struct Biol 15:176-183.

Denker BM, Smith BL, Kuhajda FP, Agre P. 1988. Identification, purification, and partial characterization of a novel $\mathrm{Mr} 28,000$ integral membrane protein from erythrocytes and renal tubules. J Biol Chem 263:15634-15642.

Detmers FJ, de Groot BL, Muller EM, Hinton A, Konings IB, Sze M, Flitsch SL, Grubmuller H, Deen PM. 2006. Quaternary ammonium compounds as water channel blockers. Specificity, potency, and site of action. J Biol Chem 281:14207-14214.

Fu D, Libson A, Miercke LJ, Weitzman C, Nollert P, Krucinski J, Stroud RM. 2000. Structure of a glycerol-conducting channel and the basis for its selectivity. Science 290:481-486.

Fu D, Libson A, Stroud R. 2002. The structure of GlpF, a glycerol conducting channel. In Ion channels: from atomic resolution physiology to functional genomics. Chichester: John Wiley \& Sons Ltd. p. 51-61.

Gonen T, Cheng Y, Sliz P, Hiroaki Y, Fujiyoshi Y, Harrison SC, Walz T. 2005. Lipid-protein interactions in double-layered twodimensional AQP0 crystals. Nature 438:633-638.

Gonen T, Sliz P, Kistler J, Cheng Y, Walz T. 2004. Aquaporin-0 membrane junctions reveal the structure of a closed water pore. Nature 429:193-197.

Gonen T, Walz T. 2006. The structure of aquaporins. Q Rev Biophys 39:361-396.

Gorelick DA, Praetorius J, Tsunenari T, Nielsen S, Agre P. 2006. Aquaporin-11: a channel protein lacking apparent transport function expressed in brain. BMC Biochem 7:1-14.

Gorin MB, Yancey SB, Cline J, Revel JP, Horwitz J. 1984. The major intrinsic protein (MIP) of the bovine lens fiber membrane: characterization and structure based on cDNA cloning. Cell 39:49-59.

Gunnarson E, Zelenina M, Aperia A. 2004. Regulation of brain aquaporins. Neuroscience 129:947-955.

Hara-Chikuma M, Verkman AS. 2006. Physiological roles of glycerol-transporting aquaporins: the aquaglyceroporins. Cell Mol Life Sci 63:1386-1392.

Harries WE, Akhavan D, Miercke LJ, Khademi S, Stroud RM. 2004. The channel architecture of aquaporin 0 at a 2.2-A resolution. Proc Natl Acad Sci USA 101:14045-14050.

Hedfalk K, Törnroth-Horsefield S, Nyblom M, Johanson U, Kjellbom P, Neutze R. 2006. Aquaporin gating. Curr Opin Struct Biol 16:447-456.

Heller KB, Lin EC, Wilson TH. 1980. Substrate specificity and transport properties of the glycerol facilitator of Escherichia coli. J Bacteriol 144:274-278.

Hiroaki Y, Tani K, Kamegawa A, Gyobu N, Nishikawa K, Suzuki H, Walz T, Sasaki S, Mitsuoka K, Kimura K, Mizoguchi A, Fujiyoshi Y. 2006. Implications of the aquaporin-4 structure on array formation and cell adhesion. J Mol Biol 355:628-639.

Ikeda M, Beitz E, Kozono D, Guggino WB, Agre P, Yasui M. 2002. Characterization of aquaporin-6 as a nitrate channel in mammalian cells. Requirement of pore-lining residue threonine 63. J Biol Chem 277:39873-39879.

Ishibashi K. 2006. Aquaporin subfamily with unusual NPA boxes. Biochim Biophys Acta 1758:989-993.

Ishibashi K, Kuwahara M, Gu Y, Kageyama Y, Tohsaka A, Suzuki F, Marumo F, Sasaki S. 1997. Cloning and functional 
expression of a new water channel abundantly expressed in the testis permeable to water, glycerol, and urea. J Biol Chem 272:20782-20786

Jiang J, Daniels BV, Fu D. 2006. Crystal structure of AqpZ tetramer reveals two distinct Arg-189 conformations associated with water permeation through the narrowest constriction of the water-conducting channel. J Biol Chem 281:454-460.

Johanson U, Karlsson M, Johansson I, Gustavsson S, Sjovall S, Fraysse L, Weig AR, Kjellbom P. 2001. The complete set of genes encoding major intrinsic proteins in Arabidopsis provides a framework for a new nomenclature for major intrinsic proteins in plants. Plant Physiol 126:1358-1369.

Johansson I, Karlsson M, Shukla VK, Chrispeels MJ, Larsson C, Kjellbom P. 1998. Water transport activity of the plasma membrane aquaporin PM28A is regulated by phosphorylation. Plant Cell 10:451-459.

Jung JS, Preston GM, Smith BL, Guggino WB, Agre P. 1994. Molecular structure of the water channel through aquaporin CHIP. The hourglass model. J Biol Chem 269:14648-14654.

Kaldenhoff R, Fischer M. 2006. Functional aquaporin diversity in plants. Biochim Biophys Acta 1758:1134-1141.

Kistler J, Bullivant S. 1980. Lens gap junctions and orthogonal arrays are unrelated. FEBS Lett 111:73-78.

Kozono D, Ding X, Iwasaki I, Meng X, Kamagata Y, Agre P, Kitagawa Y. 2003. Functional expression and characterization of an archaeal aquaporin. AqpM from methanothermobacter marburgensis. J Biol Chem 278:10649-10656.

Lee JK, Kozono D, Remis J, Kitagawa Y, Agre P, Stroud RM. 2005. Structural basis for conductance by the archaeal aquaporin AqpM at 1.68 A. Proc Natl Acad Sci USA 102:1893218937

Liu Z, Shen J, Carbrey JM, Mukhopadhyay R, Agre P, Rosen BP. 2002. Arsenite transport by mammalian aquaglyceroporins AQP7 and AQP9. Proc Natl Acad Sci USA 99:6053-6058.

Morishita Y, Sakube Y, Sasaki S, Ishibashi K. 2004. Molecular mechanisms and drug development in aquaporin water channel diseases: aquaporin superfamily (superaquaporins): expansion of aquaporins restricted to multicellular organisms. J Pharmacol Sci 96:276-279.

Murata K, Mitsuoka K, Hirai T, Walz T, Agre P, Heymann JB, Engel A, Fujiyoshi Y. 2000. Structural determinants of water permeation through aquaporin-1. Nature 407:599-605.

Nielsen S, Frøkiaer J, Marples D, Kwon TH, Agre P, Knepper MA. 2002. Aquaporins in the kidney: from molecules to medicine. Physiol Rev. 82:205-244.

Pao GM, Wu LF, Johnson KD, Hofte H, Chrispeels MJ, Sweet G, Sandal NN, Saier MH Jr. 1991. Evolution of the MIP family of integral membrane transport proteins. Mol Microbiol 5:33-37.

Park JH, Saier MH Jr. 1996. Phylogenetic characterization of the MIP family of transmembrane channel proteins. J Membr Biol 153:171-180.

Pohl P, Saparov SM, Borgnia MJ, Agre P. 2001. Highly selective water channel activity measured by voltage clamp: analysis of planar lipid bilayers reconstituted with purified AqpZ. Proc Natl Acad Sci USA 98:9624-9629.

Pomes R, Roux B. 1996. Structure and dynamics of a proton wire: a theoretical study of $\mathrm{H}+$ translocation along the single-file water chain in the gramicidin A channel. Biophys J 71:19-39.

Prasad GV, Coury LA, Finn F, Zeidel ML. 1998. Reconstituted aquaporin 1 water channels transport $\mathrm{CO} 2$ across membranes. J Biol Chem 273:33123-33126.

Preston GM, Carroll TP, Guggino WB, Agre P. 1992. Appearance of water channels in Xenopus oocytes expressing red cell CHIP28 protein. Science 256:385-387.
Preston GM, Jung JS, Guggino WB, Agre P. 1993. The mercurysensitive residue at cysteine 189 in the CHIP28 water channel. J Biol Chem 268:17-20.

Preston GM, Jung JS, Guggino WB, Agre P. 1994. Membrane topology of aquaporin CHIP. Analysis of functional epitopescanning mutants by vectorial proteolysis. J Biol Chem 269:1668-1673.

Ren G, Reddy VS, Cheng A, Melnyk P, Mitra AK. 2001. Visualization of a water-selective pore by electron crystallography in vitreous ice. Proc Natl Acad Sci USA 98: 1398-1403.

Roy D, Spector A, Farnsworth PN. 1979. Human lens membrane: comparison of major intrinsic polypeptides from young and old lenses isolated by a new methodology. Exp Eye Res 28:353-358.

Saparov SM, Liu K, Agre P, Pohl P. 2007. Fast and selective ammonia transport by aquaporin-8. J Biol Chem 282:52965301.

Saparov SM, Tsunoda SP, Pohl P. 2005. Proton exclusion by an aquaglyceroprotein: a voltage clamp study. Biol Cell 97:545550.

Savage DF, Egea PF, Robles-Colmenares Y, O'Connell JD 3rd, Stroud RM. 2003. Architecture and selectivity in aquaporins: 2.5 a X-ray structure of aquaporin Z. PLoS Biol 1:E72.

Smith BL, Agre P. 1991. Erythrocyte Mr 28,000 transmembrane protein exists as a multisubunit oligomer similar to channel proteins. J Biol Chem 266:6407-6415.

Sui H, Han BG, Lee JK, Walian P, Jap BK. 2001. Structural basis of water-specific transport through the AQP1 water channel. Nature 414:872-878.

Tajkhorshid E, Nollert P, Jensen MO, Miercke LJ, O'Connell J, Stroud RM, Schulten K. 2002. Control of the selectivity of the aquaporin water channel family by global orientational tuning. Science 296:525-530.

Tornroth-Horsefield S, Wang Y, Hedfalk K, Johanson U, Karlsson M, Tajkhorshid E, Neutze R, Kjellbom P. 2006. Structural mechanism of plant aquaporin gating. Nature 439:688-694.

Tournaire-Roux C, Sutka M, Javot H, Gout E, Gerbeau P, Luu DT, Bligny R, Maurel C. 2003. Cytosolic pH regulates root water transport during anoxic stress through gating of aquaporins. Nature 425:393-397.

Tsukaguchi H, Shayakul C, Berger UV, Mackenzie B, Devidas S, Guggino WB, van Hoek AN, Hediger MA. 1998. Molecular characterization of a broad selectivity neutral solute channel. J Biol Chem 273:24737-24743.

Wallace IS, Roberts DM. 2005. Distinct transport selectivity of two structural subclasses of the nodulin-like intrinsic protein family of plant aquaglyceroporin channels. Biochemistry 44:16826-16834.

Wang Y, Schulten K, Tajkhorshid E. 2005. What makes an aquaporin a glycerol channel? A comparative study of AqpZ and GlpF. Structure 13:1107-1118.

Yasui M. 2004. Molecular mechanisms and drug development in aquaporin water channel diseases: structure and function of aquaporins. J Pharmacol Sci 96:260-263.

Yasui M, Hazama A, Kwon TH, Nielsen S, Guggino WB, Agre P. 1999. Rapid gating and anion permeability of an intracellular aquaporin. Nature 402:184-187.

Zampighi GA, Hall JE, Ehring GR, Simon SA. 1989. The structural organization and protein composition of lens fiber junctions. J Cell Biol 108:2255-2275.

Zeidel ML, Ambudkar SV, Smith BL, Agre P. 1992. Reconstitution of functional water channels in liposomes containing purified red cell CHIP28 protein. Biochemistry 31:74367440 . 